\title{
Validity of a Novel Body Surface Electrocardiograph Mapping Device by Medtronic ${ }^{\mathrm{TM}}$
}

\author{
Seyed Ahmad Pour Hakimi 1, * \\ 1 Otto-von-Guericke University, Magdeburg, Universitatsplatz, D-39106 Magdeburg, Germany; \\ s.pourhakimi@gmail.com \\ * Author to whom correspondence should be addressed.
}

\begin{abstract}
:
Despite the 12-lead electrocardiogram (ECG) being the gold standard in evaluating clinical presentations of arrythmias of various kinds, several notable limitations, namely in accuracy and localization, have recently garnered concern with respect to administering appropriate therapeutic measures. The following text puts these limitations under the spotlight, highlighting the difficulty and challenging nature of standard ECG analysis and therapeutic measures, while simultaneously showing a novel body surface electrocardiographic mapping system that utilizes 3D spatial imaging and pinpoint localization algorithms in order to compensate for the shortcomings of the standard ECG, thereby providing higher precision data collection. We provide a brief overview of the novel device in a selection of arrhythmic demographics, along with its validity and reliability, while illustrating the benefits and positive outcomes of its utility.
\end{abstract}

Keywords: 12-lead electrocardiogram, body surface cardiac mapping, Medtronic, electrocardiograph mapping, arrythmia

\section{Introduction}

Although not entirely similar to the severity of the pandemics throughout history, cardiovascular disease (CVD) has been the leading cause of death for some time now (Sidney, Quesenberry, Jaffe, et al., 2016). With such a position amongst diseases, CVD imposes severe burden and disability on the population. Medical tools and procedures have been developed to attain preventative methods that can help reduce the negative implications CVD has on the world. One such tool, now in use for over 100 years, is the 12-lead electrocardiograph (ECG), which has established itself as the "gold standard" of the noninvasive diagnostic tool in addressing heart disease, specifically arrhythmias and conduction pathologies. The ability to obtain numerical readings of electrical measurements by the heart has long helped healthcare professionals prevent and treat heart disease. Despite being a powerful diagnostic tool, recent studies have shown that the 12-lead ECG contains several limitations that can hinder the diagnostic processes. The current pool of research has established that the 12-lead ECG lacks accuracy in detecting the location of arrhythmias, which leads to a challenging and more difficult diagnosis when it comes to assessing the efficacy of potential treatment, especially when cardio resynchronization therapy (CRT) is involved (Cochet et al., 2014; Shah, Hocini, Pascale, et al., 2013). Other studies have discussed further limitation of the 12-lead ECG including limited and general measurement of ventricular electrical activation disorders (Cakulev et al., 2013; Ploux et al., 2013) inadequately pinpointing ventricular tachycardia (VT) location (Jamil-Copley et al., 2014), and poor spatial resolution (Aryana, O'Neill, \& d'Avila, 2015). The standard feature among the presented studies demonstrates the lack of accuracy by the 12-lead ECG in determining several characteristics of heart disease, more importantly, arrhythmias, which are crucial for diagnosis. One proposed reason for such a trait lies within the ECG's algorithm. A handful of ECG algorithms have been implemented to predict arrhythmic 
activation sites, yet the reliability of each are weak and absent (Aryana et al., 2015). Irrespective of the reasons, the collective agreement amongst recent studies has concluded that the 12-lead ECG may not be as effective as once thought when it comes to localizing arrhythmia. Nevertheless, a novel tool has surfaced that has shown potential in assessing the limitations of the 12-lead ECG. The electrocardiographic mapping (ECM) method is a non-invasive, 252-lead ECG-type imaging tool that has shown accuracy in detecting a broad spectrum of arrhythmic characteristics (Shah, Hocini, Pascale, et al., 2013). The implications of this novel method have the potential to address the limitations faced by the 12-lead ECG, and more importantly, benefit the therapeutic approach to heart disease. Hereinbelow, the validity, reliability, and overall feasibility of the ECM will be addressed using the available pool of literature on a diverse selection of arrhythmias.

\section{Atrial Arrythmias \& ECM}

Despite the 12-lead ECG being limited, it still holds a high degree of validity. However, the main concern is not with the ECG, but with what the ECG tends to lead to. Because of the limitations discussed above, physicians necessitate undergoing invasive electrophysiological studies to better understand the patients underlying problems. Thus, information not obtained by the ECG must be obtained invasively.

To understand the challenging position faced by doctors investigating treatment options, or simply obtaining data, it's important to understand some of the mechanism of cardiac arrhythmias. Blood supply to tissues and organs, in part, relies on a synchronized and organized beating pattern. The way the heart beats so regularly is by electrical activation, regulated by automaticity of the biological pacemakers within the heart, and any disturbances to that pattern leads to irregularities and arrhythmias (Tse, 2016). In the event of disturbances to regular pattern, a handful of arrhythmias can surface. Issues within the atrium can cause the sinoatrial node (SA) to malfunction, and produce delayed or enhanced automaticity, causing either bradycardia or tachycardia respectively. Conduction blocks can obstruct electrical activation between the upper and lower chambers of the heart, causing each to beat to their natural pattern. Moreover, some of the arrhythmias are further distinguished by their activation patterns. In addition to that, each arrhythmia has its own unique activation pattern across the epicardium, and each has a specific localization origin (Antzelevitch \& Burashnikov, 2011). Because of the complex nature of arrhythmias, combined with the limitations of the ECG, invasive procedures must be used in order to obtain accurate information before proceeding with a therapeutic method. Putting patients through invasive procedures should be used as a last option, especially when modern tools have advanced and overcome limitations. The ECM has the ability to obtain information just as well, if not better, than invasive electrophysiological study, with the advantage of being non-invasive. The ECM's role is providing a mapping system that stores and displays electrophysiological data for analysis. The patient wears a vest consisting of 252 electrodes connected to a mapping system, which records the electrical signals.

A CT scan is used to display a 3D image of the heart. Using mathematical algorithms, the system can provide a 3D reconstructed image of the heart along with the location of the electrodes, while also providing a beat per beat activation map (Jamil-Copley et al., 2014). Being able to extract data noninvasively is a high leap in the field of diagnostics. Research has shown that ECM mapping system can effectively deliver better information and data, even with normal cardiac physiology (Oster, Taccardi, Lux, Ershler, \& Rudy, 1997). Thus, the below research takes into account the different complex types of arrhythmias, and illustrate how the ECM can deliver information accurately that can better predict therapeutic approach.

To establish ECM's efficiency in clinical practice and as a diagnostic tool, several studies have tested the method on a variety of arrhythmias. Of all the arrhythmias present, atrial arrhythmias are the most common amongst the population (Wyndham, 2000). Being as such, therapeutic approach to this common disease is of importance. Several studies have investigated and compared the mapping system to that of the standard technique, i.e. 12-lead ECG. Three studies examined the alternative novel non-invasive ECM and its role in assisting the diagnosis of atrial arrhythmias compared to the standard 12-lead ECG (Cakulev et al., 2013; Cochet et al., 2014; Shah et al., 2013). Two studies 
examined participants with atrial tachycardia (AT) (Cakulev et al., 2013; Shah et al., 2013); while one study examined participants with atrial fibrillation (AF) (Cochet et al., 2014). All three studies obtained 3D mapping sequences to assess the accuracy against invasive ablation procedures. Of the two studies that examined participants with AT, one study $(\mathrm{N}=52)$ reported a near successful association between ECM results and invasive procedures in detecting the site of arrhythmia in 48 participants (Shah et al., 2013), while the other study $(\mathrm{N}=10)$ reported a $100 \%$ success rate in localization of atrial arrhythmia (Cakulev et al., 2013). The study by Shah et al. (2013) showed that ECM identified the participants AT feature as re-entrant $(85 \%)$ or centrifugal $(100 \%)$, bringing the total evaluable number of participants (44 out of 48 ) to $92 \%$ overall accuracy.

Moreover, the ECM accurately identified all of the centrifugal AT as right atrium source or left atrium source. A further comparison taken into consideration evaluated ECM diagnostic accuracy between participants who had previous AF ablation and participants who were undergoing (firsttime) AF ablation. The results showed that overall efficiency of the ECM in diagnosing either was 92\%; the past AF ablation participants having 83\% accuracy and the first-time ablation participants having 100\% accuracy. Similarly, the second study by Cakulev et al. (2013) also reported results along the same lines. Their research also noted that the ECM technique was able to identify all their participants AT, while also differentiating between left and right atrium sources and between focal and re-entrant mechanisms. The third study by Cochet et al. (2014), consisting of AF participants, also reported successful detection of rotor activity on the epicardium and accurately detecting core location of trajectories. To supplement this notion, ECM established the ability to map epicardial activation patterns specific to the patient, which signifies both the complexity and depth of AF and the productive role of ECM as a non-invasive technique (Mason, 2009).

It is evident from the studies described here that ECM has strong traits in validation and reliability when it comes to its role in diagnostic methods. The ECM, as a result of much research and development, achieved its goal in measuring and accurately depicting atrial activation sites, rendered by a mapping system, and consisting of beat-by-beat tracking. Moreover, has enhanced the ability to differentiate atrial arrhythmia types, whether they are focal or re-entrant, and whether or not they originate in the left or right atrium. Furthermore, these results have been reproduced among several other studies, hinting towards a robust, reliable technique, which favors ECM as a suitable alternative to modern diagnostic methods.

\section{Ventricular Arrythmias \& ECM}

ECM's effectiveness also extends to ventricular arrhythmias, more notably ventricular tachycardia, which comes in varying degrees and characteristics. This type of tachycardia is particular when it comes to locating activation sites (Wang et al., 2012). Much like atrial arrhythmias, several studies have investigated ECM's role in accurately providing mapping sequences of ventricular activation sites. Of the below-mentioned studies, two included participants with WolffParkinson-White (WPW) syndrome (Cakulev et al., 2013; Cochet et al., 2014), one study included patients with premature ventricular complexes (PVC) (Jamil-Copley et al., 2014), and one study examined traits of QRS duration and left bundle branch block (LBBB) compared to ECM in predicting CRT outcomes (Ploux et al., 2013).

The studies that examined participants with WPW syndrome approached the disease mechanism differently. One of the studies used ECM to predict if the ventricular insertion fell to the right or left of the ventricular septum (Cakulev et al., 2013), while the second study used ECM to predict the localization of essential accessory pathways and sites of ventricular pre-excitation (Cochet et al., 2014). Both studies reported ECM accuracy in predicting the mechanisms respectively. The ECM technique was able to differentiate between left and right ventricular insertion, while also mapping accessory pathways and localizing pre-excitation sites. This adds to ECM's validity and reliability, in such that it was accurately able to provide the details that other methods would otherwise fail to collect.

The study that investigated PVCs sought to address the ability of ECM to predict which ventricular chamber PVC originated from. Jamil-Copley et al. (2014) reported that ECM was able to 
predict the origin chambers in $96 \%$ of the participants (23 out of 24 participants), followed by $100 \%$ localization of specific regions within the specified chamber. The implications of this are twofold. Firstly, the study went on to compare localization ability between three ECG algorithms and the ECM technique. Compared to the reported results of the ECM, which were near perfect, the combined effectiveness of the three algorithms predicted between $50 \%-80 \%$ the correct location, i.e. chamber, of PVC, and in addition to that, when the proper chamber was located, a mere $37 \%-58 \%$ predicted the right region within the chamber. Being able to distinguish correctly between chambers leads to the second implication, which is concerned with the ECM being used as a guide during invasive procedures such as catheter ablation. Thus, not only does the ECM serve as a diagnostic tool in accurately predicting the location of ventricular arrhythmias, but it also serves as a guide to successful ablation procedures. In addition to this notion, ECM is seen to extend its role in CRT as well. The study by Ploux et al. (2013) aimed at addressing the effectiveness of ECM in predicting successful outcomes when applying for CRT, compared to QRS length. This was done by measuring ventricular activation times in patients with QRS of $120 \mathrm{~ms}$ or more. It was reported that using ECM to establish ventricular electrical uncoupling (VEU), which is the difference between LV activation time and RV activation time, provided a much more accurate picture for CRT response compared to 12-lead ECG predictor of QRS length. In supplement to this study, Eschalier et al. (2015) also noted the useful role ECM has in providing better insight into ventricular activation times, especially when it came to CRT response. It is still a challenge to understand why some patients are more likely to benefit from CRT than others; there is innovative research focusing on intraventricular delay patterns, indexes of QRS prolongation and ventricular activation desynchrony as to provide a reason why. In light of understanding further mechanism insights, ECM can give detailed information about the therapeutic response. Mainly ECM can provide information that can be used to reason whatever CRT would benefit specific people. The significance of this, adding to the two-fold implications discussed earlier, is that the ECM can also provide accurate data that can gravitate to a healthcare professional decision when it comes to the therapeutic approach. Being able to predict whether a patient will benefit from CRT is a valuable asset. The study conducted by Woodard \& Rudy (2010) exemplifies the necessity of having accurate data to predict CRT outcomes. In this study, the participants consisted of pediatrics with heart failure, which were in the process of obtaining some form of CRT. The mechanism studied was ventricular electrical desynchrony (ED), and was established to be typically $20+/-4$ ms. Divided into two groups of responders and non-responders to CRT, the study confirmed that the responder group had and ED index of $22 \mathrm{~ms}$ compared to their counterpart group, who showed ED values averaging $37 \mathrm{~ms}$. Thus, ECM was able to provide data determining which patients would benefit from CRT. A final trait that ECM puts forward is the ability to guide lead placement during implantation. This is so because the mapping system can pinpoint electrical activation sites accurately, showing where the last excitation area was.

12-lead ECG classification is the fundamental tool for classification of arrhythmias. We know now, from the studies addressed above, that a new mapping technique of ECM can "upgrade" the designation of arrhythmias that was once given by 12-lead ECGs. Such a notion can be established because the ECM has demonstrated high validity and reliability across a handful of studies. The mapping system was tasked and used to measure a wide range of arrhythmias, including AF and VF, PVCs, and WPW syndrome, all of which is accurately defined and reported. Moreover, ECM was used to determine which patients will be likely to respond to CRT. Not only does ECM's accuracy earn its validation, but its reproducibility amongst many other studies also gains its right to reliability. Not every study reported examined the same number of patients using the same method, but their results were consistent with each other, which makes the ECM highly effective in diagnostic practices. The above studies reported that one of ECM's features was to report electrical activity and excitation times along the epicardium by measuring potentials, which in effect, could render a 3D map of the heart (Rudy \& Burnes, 1999).

\section{Summary}


It's evident that ECM has shown promise and effectiveness in areas where the 12-lead ECG has displayed limitations. The above studies demonstrate a unifying aspect of the ECM as being a noninvasive mapping tool that can detect arrhythmic activation sites and accurately locate their origin, a feature not found in the standard care methods today. Moreover, the ECM demonstrated the ability not only to localize atrial and ventricular arrhythmias, but also to differentiate their types, whether they are focal or re-entrant, and whether or not they originate in the left or right atrium/ventricle. The ECM can provide the healthcare professional with ample information and data that can help with the therapeutic approach, all done non-invasively, which in effect can, as we have seen in the studies above, result in higher success rates during invasive procedures such as ablations. The potential of ECM has yet to be fulfilled, and when done so, clinical diagnostic methods will witness a great bump upwards.

\section{Conflicts of Interest:}

The author declares no conflict of interest.

\section{References}

1. Antzelevitch, C., \& Burashnikov, A. (2011). Overview of basic mechanism of cardiac arrhythmia. Card Electrophysiol Clin., 3(1), 23-45. doi.org/10.1016/j.ccep.2010.10.012.Overview

2. Aryana, A., O'Neill, P. G., \& d'Avila, A. (2015). Noninvasive electrocardiographic mapping: Are we ready for prime time? Journal of the American Heart Association, 4(10), 1-4. doi.org/10.1161/JAHA.115.002655

3. Cakulev, I., Sahadevan, J., Arruda, M., Goldstein, R. N., Hong, M., Intini, A., ... Waldo, A. L. (2013). Confirmation of novel noninvasive high-density electrocardiographic mapping with electrophysiology study: Implications for therapy. Circulation: Arrhythmia and Electrophysiology, 6(1), 68-75. doi.org/10.1161/CIRCEP.112.975813

4. Cochet, H., Dubois, R., Sacher, F., Derval, N., Sermesant, M., Hocini, M., ... Jaïs, P. (2014). Cardiac Arrythmias: Multimodal Assessment Integrating Body Surface ECG Mapping into Cardiac Imaging. Radiology, 271(1), 239-247. doi.org/10.1148/radiol.13131331

5. Jamil-Copley, S., Bokan, R., Kojodjojo, P., Qureshi, N., Koa-Wing, M., Hayat, S., ... Lim, P. B. (2014). Noninvasive electrocardiographic mapping to guide ablation of outflow tract ventricular arrhythmias. Heart Rhythm, 11(4), 587-594. doi.org/10.1016/j.hrthm.2014.01.013

6. Mason, A. (2009). NIH Public Access, 19(14), 389-399. doi.org/10.1016/j.asieco.2008.09.006.EAST

7. Oster, H. S., Taccardi, B., Lux, R. L., Ershler, P. R., \& Rudy, Y. (1997). Noninvasive Electrocardiographic Imaging. Circulation, 96(3), 1012-1024. doi.org/10.1161/01.cir.96.3.1012

8. Ploux, S., Lumens, J., Whinnett, Z., Montaudon, M., Strom, M., Ramanathan, C., ... Bordachar, P. (2013). Noninvasive Electrocardiographic Mapping to Improve Patient Selection for Cardiac Resynchronization Therapy. Journal of the American College of Cardiology, 61(24), 2435-2443. doi.org/10.1016/j.jacc.2013.01.093

9. Rudy, Y., \& Burnes, J. E. (1999). Noninvasive electrocardiographic imaging. Annals of Noninvasive Electrocardiology, 4(3), 340-359. doi.org/10.1111/j.1542-474X.1999.tb00220.x

10. Shah, A. J., Hocini, M., Pascale, P., Roten, L., Komatsu, Y., Daly, M., ... Haissaguerre, M. (2013). Body Surface Electrocardiographic Mapping for Non-invasive Identification of Arrhythmic Sources. Arrhythmia \& Electrophysiology Review, 2(1), 16. doi.org/10.15420/aer.2013.2.1.16

11. Shah, A. J., Hocini, M., Xhaet, O., Pascale, P., Roten, L., Wilton, S. B., ... Haissaguerre, M. (2013). Validation of novel 3-dimensional electrocardiographic mapping of atrial tachycardias by invasive mapping and 
ablation: A multicenter study. Journal of the American College of Cardiology, 62(10), 889-897. doi.org/10.1016/j.jacc.2013.03.082

12. Tse, G. (2016). Mechanisms of cardiac arrhythmias. Journal of Arrhythmia, 32(2), $75-81$. doi.org/10.1016/j.joa.2015.11.003

13. Wang, Y., Cuculich, P. S., Zhang, J., Desouza, K. A., Smith, T. W., \& Rudy, Y. (2012). NIH Public Access, 3(98). doi.org/10.1126/scitranslmed.3002152.Noninvasive

14. Wyndham, C. R. (2000). Atrial fibrillation: the most common arrhythmia. Texas Heart Institute Journal / from the Texas Heart Institute of St. Luke's Episcopal Hospital, Texas Children's Hospital, 27(3), 257-267. doi.org/10.4061/2011/837841

15. Sidney, S., Quesenberry, C. P., Jaffe, M. G., Sorel, M., Nguyen-Huynh, M. N., Kushi, L. H., . . Rana, J. S. (2016). Recent Trends in Cardiovascular Mortality in the United States and Public Health Goals. JAMA Cardiology, 1(5), 594. doi:10.1001/jamacardio.2016.1326

16. Ramanathan, C., Ghanem, R. N., Jia, P., Ryu, K., \& Rudy, Y. (2004). Noninvasive electrocardiographic imaging for cardiac electrophysiology and arrhythmia. Nature Medicine, 10(4), 422-428. doi.org/10.1038/nm1011 